Pacific

Journal of

Mathematics

\title{
THE DISCRIMINANT OF A SYMPLECTIC INVOLUTION
}

Grégory Berhuy, Marina Monsurrò, and Jean-Pierre Tignol 


\title{
THE DISCRIMINANT OF A SYMPLECTIC INVOLUTION
}

\author{
Grégory Berhuy, Marina Monsurrò, and Jean-Pierre Tignol
}

An invariant for symplectic involutions on central simple algebras of degree divisible by 4 over fields of characteristic different from 2 is defined on the basis of Rost's cohomological invariant of degree 3 for torsors under symplectic groups. We relate this invariant to trace forms and show how its triviality yields a decomposability criterion for algebras of degree 8 with symplectic involution.

\section{Introduction and statement of results.}

In contrast with orthogonal involutions, for which invariants corresponding to the discriminant and Clifford algebras of quadratic forms are defined, no "classical" invariant is known for symplectic involutions on central simple algebras, besides the signature (see $[\mathbf{6},(11.10)]$ ). Using the cohomological invariant of degree 3 defined by Rost for torsors under simply connected absolutely simple linear algebraic groups, we introduce an invariant of symplectic involutions on central simple algebras of degree a multiple of 4 with values in the third Galois cohomology group of the center with coefficients $\{ \pm 1\}$ and give an alternative description in terms of trace forms. We call this invariant the discriminant since it is the first nontrivial invariant, and because it is directly linked to the discriminant of Hermitian forms, see Example 2. Even though its definition is elementary, Rost's computation of the invariants of torsors under symplectic groups is needed to prove that there is no other cohomological invariant of degree 3 and to establish the relationship with trace forms. In the final section, we prove that symplectic involutions with trivial discriminant on central simple algebras of degree 8 and index 4 afford a special type of decomposition. In a sequel to this paper, the discriminant is used to give examples of non $R$-trivial adjoint symplectic groups of even index.

1.1. Definition of the discriminant. Throughout this paper, $F$ denotes a field of characteristic different from 2. Let $A$ be a finite-dimensional central simple $F$-algebra, and $\theta: A \rightarrow A$ be an anti-automorphism of order 2 . We recall that $\theta$ is called a symplectic involution on $A$ if, after scalar extension to a splitting field, $\theta$ is adjoint to an alternating form, see [6, (2.5)]. From now on, we suppose the involution $\theta$ is of this type. In this case, the degree $\operatorname{deg} A$ is necessarily an even integer $n=2 m$. 
The symplectic group $\mathbf{S p}(A, \theta)$ is the group scheme over $F$ defined by

$$
\mathbf{S p}(A, \theta)(E)=\left\{x \in A \otimes_{F} E \mid \theta(x) x=1\right\}
$$

for any commutative $F$-algebra $E$.

Let $\operatorname{Sym}(A, \theta)$ be the $F$-vector space of elements in $A$ fixed by $\theta$,

$$
\operatorname{Sym}(A, \theta)=\{x \in A \mid \theta(x)=x\} .
$$

We denote by $\operatorname{Sym}(A, \theta)^{\times}$the set of units in $\operatorname{Sym}(A, \theta)$,

$$
\operatorname{Sym}(A, \theta)^{\times}=\operatorname{Sym}(A, \theta) \cap A^{\times} .
$$

We recall that the pfaffian reduced norm is the homogeneous polynomial function of degree $m$

$$
\operatorname{Nrp}_{\theta}: \operatorname{Sym}(A, \theta) \rightarrow F
$$

uniquely determined by the following conditions:

$$
\operatorname{Nrp}_{\theta}(1)=1 \quad \text { and } \quad \operatorname{Nrp}_{\theta}(x)^{2}=\operatorname{Nrd}_{A}(x) \quad \text { for } x \in \operatorname{Sym}(A, \theta),
$$

see $[6$, p. 19].

The cohomology set $H^{1}(F, \mathbf{S p}(A, \theta))$ can be represented as

$$
H^{1}(F, \mathbf{S p}(A, \theta)) \simeq \operatorname{Sym}(A, \theta)^{\times} / \sim
$$

where $\sim$ is the equivalence relation defined by $x \sim y$ if and only if there exists $u \in A^{\times}$such that $y=u x \theta(u)$, see $[\mathbf{6},(29.24)]$.

Let $\mathbf{G}_{\mathbf{m}}$ be the multiplicative group. The Kummer exact sequence

$$
1 \rightarrow \mu_{2} \rightarrow \mathbf{G}_{\mathbf{m}} \stackrel{2}{\rightarrow} \mathbf{G}_{\mathbf{m}} \rightarrow 1
$$

allows us to identify the cohomology sets $H^{1}\left(F, \mu_{2}\right)$ and $H^{2}\left(F, \mu_{2}\right)$ respectively with the quotient $F^{\times} / F^{\times 2}$ and with the 2-torsion subgroup of the Brauer group. For all $x \in F^{\times}$, we denote by $(x)_{2} \in H^{1}\left(F, \mu_{2}\right)$ the cohomology class associated to $x F^{\times 2}$. Similarly, we denote by $[A] \in H^{2}\left(F, \mu_{2}\right)$ the cohomology class associated to the Brauer class of $A$. We define

$$
\Delta_{\theta}: \operatorname{Sym}(A, \theta)^{\times} \rightarrow H^{3}\left(F, \mu_{2}\right)
$$

as the map given by the cup-product

$$
\Delta_{\theta}(s)=\left(\operatorname{Nrp}_{\theta}(s)\right)_{2} \cup[A] .
$$

It follows from the properties of $\operatorname{Nrp}_{\theta}$ (see the proof of Proposition 1 below) that $\Delta_{\theta}$ is well-defined on the set of equivalence classes under the relation $\sim$. The induced map on the quotient can be interpreted under the bijection (1) as the Rost invariant of $H^{1}(F, \mathbf{S p}(A, \theta))$, see [6, p. 440].

Since $\operatorname{Nrp}_{\theta}$ is homogeneous of degree $m$, we obtain, for $\alpha \in F^{\times}$and $s \in \operatorname{Sym}(A, \theta)^{\times}$, the following relation:

$$
\Delta_{\theta}(\alpha s)=\left(\alpha^{m} \operatorname{Nrp}_{\theta}(s)\right)_{2} \cup[A]= \begin{cases}\Delta_{\theta}(s) & \text { if } m \text { is even, } \\ \Delta_{\theta}(s)+(\alpha)_{2} \cup[A] & \text { if } m \text { is odd. }\end{cases}
$$


Therefore, if $m$ is even one can define a relative invariant for symplectic involutions on $A$ as follows:

Definition. Let $A$ be a central simple algebra over $F$ of degree $n=2 m \equiv$ $0 \bmod 4$. Let $\theta$ and $\sigma$ be symplectic involutions on $A$. There exists (see $[6$, (2.7)]) $s \in \operatorname{Sym}(A, \theta)^{\times}$such that

$$
\sigma=\operatorname{Int}(s) \circ \theta
$$

where $\operatorname{Int}(s)$ denotes the inner automorphism associated with $s$,

$$
\operatorname{Int}(s)(x)=s x s^{-1} \quad \text { for } x \in A .
$$

The element $s$ is uniquely determined up to multiplication by an element of $F^{\times}$. By (2), it follows that $\Delta_{\theta}(s) \in H^{3}\left(F, \mu_{2}\right)$ only depends on $\sigma$, since $m$ is even. We call this element the discriminant of $\sigma$ with respect to $\theta$ and denote it by $\Delta_{\theta}(\sigma)$. Thus,

$$
\Delta_{\theta}(\sigma)=\left(\operatorname{Nrp}_{\theta}(s)\right)_{2} \cup[A] \in H^{3}\left(F, \mu_{2}\right) .
$$

In the case $m=2$, an analogue of this invariant has been studied in [6, $\S 16 . \mathrm{B}]$, where it is denoted by $j_{\theta}(\sigma)$. Theorem (16.19) of $[6]$ shows that this invariant classifies, up to conjugation, symplectic involutions on a central simple algebra of degree 4.

In Section 2 we establish the following elementary result:

\section{Proposition 1.}

(a) The discriminant $\Delta_{\theta}(\sigma)$ only depends on the conjugacy classes of $\theta$ and $\sigma$, namely, if $u, v \in A^{\times}$and

$$
\theta^{\prime}=\operatorname{Int}(u) \circ \theta \circ \operatorname{Int}(u)^{-1}, \quad \sigma^{\prime}=\operatorname{Int}(v) \circ \sigma \circ \operatorname{Int}(v)^{-1},
$$

then

$$
\Delta_{\theta^{\prime}}\left(\sigma^{\prime}\right)=\Delta_{\theta}(\sigma) .
$$

In particular, if $\sigma$ and $\theta$ are conjugate, then $\Delta_{\theta}(\sigma)=0$.

(b) Let $\rho, \sigma$ and $\theta$ be symplectic involutions on $A$; then

$$
\Delta_{\rho}(\sigma)=\Delta_{\rho}(\theta)+\Delta_{\theta}(\sigma) \quad \text { and } \quad \Delta_{\theta}(\sigma)=\Delta_{\sigma}(\theta) .
$$

If the Schur index ind $A$ divides $\frac{1}{2} \operatorname{deg} A$, i.e., if $A \simeq M_{2}\left(A_{0}\right)$ for some central simple $F$-algebra $A_{0}$, then $A$ carries hyperbolic symplectic involutions, such as $\gamma \otimes \theta_{0}$, where $\gamma$ is the (unique) symplectic involution on $M_{2}(F)$ and $\theta_{0}$ is an arbitrary orthogonal involution on $A_{0}$. Since all hyperbolic involutions are pairwise conjugate, we may set $\Delta=\Delta_{\theta}$ for any hyperbolic symplectic involution $\theta$.

Example 2. Consider the algebra $A=\operatorname{End}_{Q} V$, where $Q$ is a quaternion division $F$-algebra and $V$ is an $m$-dimensional $Q$-vector space. Symplectic involutions on $A$ are then adjoint to Hermitian forms on $V$ with respect to 
the conjugation involution on $Q$. Suppose that $m$ is even and let $\sigma$ be the involution adjoint to a fixed Hermitian form $h$ on $V$. Let

$$
h=\left\langle\alpha_{1}, \ldots, \alpha_{m}\right\rangle
$$

be the diagonalization of $h$ relative to some orthogonal basis $e$ of $V\left(\alpha_{1}, \ldots\right.$, $\left.\alpha_{m} \in F^{\times}\right)$, then

$$
\Delta(\sigma)=\left((-1)^{m / 2} \alpha_{1} \ldots \alpha_{m}\right)_{2} \cup[Q] .
$$

Indeed, let $\theta$ be the hyperbolic involution adjoint to the Hermitian form with diagonalization $\langle 1,-1, \ldots, 1,-1\rangle$ relative to the basis $e$. Then, identifying $A$ with $M_{m}(Q)$ by $e$, we get

$$
\sigma=\operatorname{Int} \operatorname{diag}\left(\alpha_{1},-\alpha_{2}, \ldots, \alpha_{m-1},-\alpha_{m}\right) \circ \theta,
$$

and we can compute $\Delta(\sigma)=\Delta_{\theta}(\sigma)$ by Lemma $9(\mathrm{e})$ below.

Note that if $V_{0} \subset V$ is the $F$-subspace spanned by $e$, then $A=\left(\operatorname{End}_{F} V_{0}\right) \otimes$ $Q$, and we obtain a decomposition $\sigma=\sigma_{0} \otimes \gamma$ where $\sigma_{0}$ is the involution adjoint to the bilinear form on $V_{0}$ with diagonalization $\left\langle\alpha_{1}, \ldots, \alpha_{m}\right\rangle$ relative to $e$, and $\gamma$ is the canonical (conjugation) involution on $Q$.

This example can be slightly generalized:

Example 3. Consider the algebra $A=A_{0} \otimes_{F} Q$ where $Q$ is a quaternion $F$-algebra and $A_{0}$ is a central simple $F$-algebra. Let $\sigma_{0}$ be an orthogonal involution on $A_{0}, \gamma$ the canonical involution on $Q$, and

$$
\sigma=\sigma_{0} \otimes \gamma
$$

Suppose that ind $A_{0}$ divides $\frac{1}{2} \operatorname{deg} A_{0}$. Then

$$
\Delta(\sigma)=\left(\operatorname{disc} \sigma_{0}\right)_{2} \cup[Q],
$$

where $\operatorname{disc} \sigma_{0} \in F^{\times} / F^{\times 2}$ is the discriminant of the orthogonal involution $\sigma_{0}$ (see $\left.[6, \S 7]\right)$. Indeed, let $\theta_{0}$ be a hyperbolic orthogonal involution on $A_{0}$ and let $x_{0} \in \operatorname{Sym}\left(A_{0}, \theta_{0}\right)^{\times}$be such that $\sigma_{0}=\operatorname{Int}\left(x_{0}\right) \circ \theta_{0}$. The involution $\theta=\theta_{0} \otimes \gamma$ is hyperbolic, and we have $\sigma=\operatorname{Int}\left(x_{0} \otimes 1\right) \circ \theta$, so that

$$
\Delta(\sigma)=\left(\operatorname{Nrp}_{\theta}\left(x_{0} \otimes 1\right)\right)_{2} \cup[A] .
$$

Now, by Lemma 9(d), $\operatorname{Nrp}_{\theta}\left(x_{0} \otimes 1\right)=\operatorname{Nrd}_{A_{0}}\left(x_{0}\right)$. Equation (3) follows, since disc $\sigma_{0}$ is represented by $\operatorname{Nrd}_{A_{0}}\left(x_{0}\right)$, and $\left(\operatorname{Nrd}_{A_{0}}\left(x_{0}\right)\right)_{2} \cup\left[A_{0}\right]=0$.

1.2. Trace forms. Let $A$ be an arbitrary central simple $F$-algebra. For every involution $\sigma: A \rightarrow A$, the associated trace form $T_{\sigma}: A \rightarrow F$ is defined as follows:

$$
T_{\sigma}(x)=\operatorname{Trd}_{A}(\sigma(x) x)
$$


where $\operatorname{Trd}_{A}$ denotes the reduced trace. Denote by $T_{\sigma}^{+}$the restriction of $T_{\sigma}$ to $\operatorname{Sym}(A, \sigma)$; this form can also be seen as the restriction to $\operatorname{Sym}(A, \sigma)$ of the form $T_{A}: A \rightarrow F$ defined by

$$
T_{A}(x)=\operatorname{Trd}_{A}\left(x^{2}\right) .
$$

As is the case with involutions of the other types (see $[\mathbf{6}, \S 11]$ ), the discriminant of symplectic involutions can be expressed in terms of trace forms; indeed we have the following result:

Theorem 4. Let $A$ be a central simple algebra over $F$ and let $\theta$ and $\sigma$ be symplectic involutions on $A$. The class in the Witt ring $W F$ of the difference $T_{\sigma}^{+}-T_{\theta}^{+}$lies in the third power of the fundamental ideal, namely

$$
T_{\sigma}^{+}-T_{\theta}^{+} \in I^{3} F \text {. }
$$

Moreover, if $e_{3}: I^{3} F \rightarrow H^{3}\left(F, \mu_{2}\right)$ denotes the Arason invariant, we obtain

$$
e_{3}\left(T_{\sigma}^{+}-T_{\theta}^{+}\right)=\left\{\begin{array}{lll}
\Delta_{\theta}(\sigma) & \text { if } \operatorname{deg} A \equiv 0 & \bmod 4, \\
0 & \text { if } \operatorname{deg} A \equiv 2 & \bmod 4 .
\end{array}\right.
$$

A proof of this result is given in Section 3 below. For the trace forms $T_{\sigma}$, we have the following result:

Corollary 5. Keeping the notation of the previous theorem, we have $T_{\sigma}-$ $T_{\theta} \in I^{4} F$ and

$$
e_{4}\left(T_{\sigma}-T_{\theta}\right)= \begin{cases}(-1)_{2} \cup \Delta_{\theta}(\sigma) & \text { if } \operatorname{deg} A \equiv 0 \quad \bmod 4, \\ 0 & \text { if } \operatorname{deg} A \equiv 2 \bmod 4,\end{cases}
$$

where $e_{4}: I^{4} F \rightarrow H^{4}\left(F, \mu_{2}\right)$ denotes the degree 4 invariant.

Proof. Let $T_{\sigma}^{-}$be the restriction of $T_{\sigma}$ (or of $-T_{A}$ ) to the space of skewsymmetric elements in $A$. We have

$$
T_{\sigma}=T_{\sigma}^{+}+T_{\sigma}^{-} \quad \text { and } \quad T_{A}=T_{\sigma}^{+}-T_{\sigma}^{-},
$$

so that $T_{\sigma}=2 T_{\sigma}^{+}-T_{A}$. Similarly, $T_{\theta}=2 T_{\theta}^{+}-T_{A}$, so that

$$
T_{\sigma}-T_{\theta}=2\left(T_{\sigma}^{+}-T_{\theta}^{+}\right),
$$

hence the corollary is a direct consequence of the previous theorem.

In the special case where $\theta$ is hyperbolic we get:

Proposition 6. Suppose $A=M_{2}\left(A_{0}\right)$ for some central simple F-algebra $A_{0}$, and let $\theta$ be a hyperbolic symplectic involution on $A$. Then $T_{\theta}^{+}$is Wittequivalent to $\langle 2\rangle \cdot T_{A_{0}}$, and $T_{\theta}$ is hyperbolic. If $\operatorname{deg} A \equiv 2 \bmod 4$, then $A$ is split, hence every symplectic involution on $A$ is hyperbolic. If $\operatorname{deg} A \equiv$ $0 \bmod 4$, then, for any symplectic involution $\sigma$ on $A$, we have $T_{\sigma} \in I^{4} F$ and

$$
e_{4}\left(T_{\sigma}\right)=(-1)_{2} \cup \Delta(\sigma) .
$$

The proof is at the end of Section 3. 
1.3. Decomposability of symplectic involutions. Section 4 below will be devoted to the relations between the discriminant and the decomposability of symplectic involutions as tensor products of involutions defined on subalgebras. Our main result is concerned with degree 8 algebras with index dividing 4. Such algebras can be written in the form $A=M_{2}\left(A_{0}\right)$, where $A_{0}$ is a central simple algebra of degree 4 , hence they carry hyperbolic symplectic involutions. The case ind $A=1$ is trivial, since every symplectic involution on a split algebra is hyperbolic, and is omitted in the following theorem:

Theorem 7. Let $A$ be a central simple F-algebra of degree 8 having index 2 or 4 . For any symplectic involution $\sigma$ on $A$, there is a decomposition

$$
(A, \sigma)=(Q, \gamma) \otimes_{F}\left(A_{0}, \sigma_{0}\right)
$$

where $Q$ is a quaternion subalgebra, $A_{0}$ is its centralizer (which is a central simple $F$-subalgebra of degree 4 in $A), \gamma$ is the conjugation involution on $Q$ and $\sigma_{0}$ is an orthogonal involution on $A_{0}$.

When ind $A=2$, this theorem is easily proved and can be readily generalized to any degree, see Example 2 or [1, Proposition 3.4]. The case ind $A=4$ is treated in Section 4.

Theorem 7 shows that the discriminant of a symplectic involution $\sigma$ on a central simple $F$-algebra of degree 8 and index 2 or 4 can be computed as in Example 3 above. The following theorem gives a necessary and sufficient condition for the discriminant to be trivial.

Theorem 8. Let $A$ be a central simple F-algebra of degree 8 with index dividing 4. For any symplectic involution $\sigma$ on $A, \Delta(\sigma)=0$ if and only there is a decomposition

$$
(A, \sigma)=\left(A_{1}, \sigma_{1}\right) \otimes_{F}\left(A_{2}, \sigma_{2}\right) \otimes_{F}\left(A_{3}, \gamma_{3}\right)
$$

where $A_{1}, A_{2}, A_{3}$ are quaternion subalgebras of $A, \sigma_{1}, \sigma_{2}$ are orthogonal involutions on $A_{1}$ and $A_{2}$ respectively, $\gamma_{3}$ is the conjugation involution on $A_{3}$, and $A_{1}$ is split,

$$
A_{1} \simeq M_{2}(F) .
$$

A proof is given in Section 4 .

\section{Discriminants and Pfaffian norms.}

The goal of this section is to prove Proposition 1. Throughout the section, $A$ denotes a central simple $F$-algebra of degree $n=2 m$.

Lemma 9. Let $\sigma$ and $\theta$ be symplectic involutions on $A$ and let $s$ be an element in $\operatorname{Sym}(A, \theta)^{\times}$such that $\sigma=\operatorname{Int}(s) \circ \theta$. Then: 
(a) For every $x \in \operatorname{Sym}(A, \sigma) \cap \operatorname{Sym}(A, \theta)$,

$$
\operatorname{Nrp}_{\sigma}(x)=\operatorname{Nrp}_{\theta}(x) .
$$

(b) For every $x \in \operatorname{Sym}(A, \theta)$, the product sx lies in $\operatorname{Sym}(A, \sigma)$ and

$$
\operatorname{Nrp}_{\sigma}(s x)=\operatorname{Nrp}_{\theta}(s) \operatorname{Nrp}_{\theta}(x) \text {. }
$$

(c) For every $x \in \operatorname{Sym}(A, \theta)^{\times}$,

$$
\operatorname{Nrp}_{\theta}\left(x^{-1}\right)=\operatorname{Nrp}_{\theta}(x)^{-1} .
$$

(d) Suppose $A=A_{1} \otimes_{F} A_{2}$ for some central simple $F$-algebras $A_{1}, A_{2} \subset A$ of degree $n_{1}=2 m_{1}$ and $n_{2}=2 m_{2}$ respectively; if $x_{1} \in A_{1}$ and $x_{2} \in A_{2}$ are such that $x_{1} \otimes x_{2} \in \operatorname{Sym}(A, \theta)$, then

$$
\operatorname{Nrp}_{\theta}\left(x_{1} \otimes x_{2}\right)=\operatorname{Nrd}_{A_{1}}\left(x_{1}\right)^{m_{2}} \operatorname{Nrd}_{A_{2}}\left(x_{2}\right)^{m_{1}} .
$$

(e) Suppose $A=M_{r}\left(A_{0}\right)$ and $\theta\left(\left(a_{i j}\right)_{1 \leq i, j \leq r}\right)=\left(\theta_{0}\left(a_{i j}\right)\right)_{1 \leq i, j \leq r}^{t}$ for some symplectic involution $\theta_{0}$ on the central simple F-algebra $\bar{A}_{0}$. For the diagonal matrix $x=\operatorname{diag}\left(x_{1}, \ldots, x_{r}\right)$ with $x_{i} \in \operatorname{Sym}\left(A_{0}, \theta_{0}\right)$ for $i=$ $1, \ldots, r$, we have

$$
\operatorname{Nrp}_{\theta}(x)=\operatorname{Nrp}_{\theta_{0}}\left(x_{1}\right) \ldots \operatorname{Nrp}_{\theta_{0}}\left(x_{r}\right) .
$$

Proof. (a) Let $t$ be an indeterminate over $F$. Define

$$
\operatorname{Prp}_{\sigma, x}(t)=\operatorname{Nrp}_{\sigma}(t-x) \in F[t], \quad \operatorname{Prp}_{\theta, x}(t)=\operatorname{Nrp}_{\theta}(t-x) \in F[t] .
$$

Those polynomials, called pfaffian characteristic polynomials in [6, p. 19], are monic and satisfy

$$
\operatorname{Prp}_{\sigma, x}^{2}=\operatorname{Pcrd}_{A, x}=\operatorname{Prp}_{\theta, x}^{2},
$$

where $\operatorname{Pcrd}_{A, x}(t)=\operatorname{Nrd}_{A(t)}(t-x)$ is the reduced characteristic polynomial of $x$. Therefore, $\operatorname{Prp}_{\sigma, x}(t)=\operatorname{Prp}_{\theta, x}(t)$, and evaluation at $t=0$ yields $\operatorname{Nrp}_{\sigma}(x)=\operatorname{Nrp}_{\theta}(x)$.

(b) Straightforward calculations show that $\sigma(s x)=s x$ if $\theta(x)=x$. Let us consider the two sides of the equality we aim to prove as polynomial functions of $x$. The squares of the two sides are equal since the reduced norm is multiplicative, hence they are equal up to sign. Moreover, they are equal and nonzero for $x=1$ in view of Part (a). Hence, they are equal for all $x$.

(c) We apply (b) with $x=s^{-1}$ and use the relation $\operatorname{Nrp}_{\sigma}(1)=1$.

(d) By taking the square root on both sides of the equation

$$
\operatorname{Pcrd}_{A, x_{1} \otimes x_{2}}=\left(\operatorname{Pcrd}_{A_{1}, x_{1}}\right)^{n_{2}}\left(\operatorname{Pcrd}_{A_{2}, x_{2}}\right)^{n_{1}},
$$

we obtain

$$
\operatorname{Prp}_{\theta, x_{1} \otimes x_{2}}=\left(\operatorname{Pcrd}_{A_{1}, x_{1}}\right)^{m_{2}}\left(\operatorname{Pcrd}_{A_{2}, x_{2}}\right)^{m_{1}} .
$$

The property follows by considering the constant terms. 
(e) As in the preceding case, the property follows by extracting the monic square root of each side of the equation

$$
\operatorname{Pcrd}_{A, x}=\operatorname{Pcrd}_{A_{0}, x_{1}} \ldots \operatorname{Pcrd}_{A_{0}, x_{r}} .
$$

Proposition 1 easily follows from the lemma above. Indeed, if $\sigma=\operatorname{Int}(s) \circ$ $\theta$, so that $\Delta_{\theta}(\sigma)=\left(\operatorname{Nrp}_{\theta}(s)\right)_{2} \cup[A]$, then $\theta=\operatorname{Int}\left(s^{-1}\right) \circ \sigma$ and hence $\Delta_{\sigma}(\theta)=\left(\operatorname{Nrp}_{\sigma}\left(s^{-1}\right)\right)_{2} \cup[A]$. Now Lemma 9 shows that

$$
\operatorname{Nrp}_{\sigma}\left(s^{-1}\right)=\operatorname{Nrp}_{\theta}(s)^{-1},
$$

hence $\left(\operatorname{Nrp}_{\sigma}\left(s^{-1}\right)\right)_{2}=\left(\operatorname{Nrp}_{\theta}(s)\right)_{2}$, and so $\Delta_{\sigma}(\theta)=\Delta_{\theta}(\sigma)$. If $\rho$ is another symplectic involution, and if $t \in \operatorname{Sym}(A, \rho)$ is such that $\theta=\operatorname{Int}(t) \circ \rho$, then $\sigma=\operatorname{Int}(s t) \circ \rho$. Part (b) of Lemma 9 yields

$$
\operatorname{Nrp}_{\sigma}(s t)=\operatorname{Nrp}_{\theta}(s) \operatorname{Nrp}_{\theta}(t) .
$$

Moreover, Part (a) shows that $\operatorname{Nrp}_{\sigma}(s t)=\operatorname{Nrp}_{\rho}(s t)$ and $\operatorname{Nrp}_{\theta}(t)=\operatorname{Nrp}_{\rho}(t)$. Therefore, the preceding equality can be written as

$$
\operatorname{Nrp}_{\rho}(s t)=\operatorname{Nrp}_{\theta}(s) \operatorname{Nrp}_{\rho}(t) .
$$

It follows that

$$
\begin{aligned}
\Delta_{\rho}(\sigma) & =\left(\operatorname{Nrp}_{\rho}(s t)\right)_{2} \cup[A] \\
& =\left(\operatorname{Nrp}_{\theta}(s)\right)_{2} \cup[A]+\left(\operatorname{Nrp}_{\rho}(t)\right)_{2} \cup[A]=\Delta_{\theta}(\sigma)+\Delta_{\rho}(\theta),
\end{aligned}
$$

which completes the proof of Part (b) of Proposition 1.

Now let $v \in A^{\times}$and $\sigma^{\prime}=\operatorname{Int}(v) \circ \sigma \circ \operatorname{Int}(v)^{-1}$, so that $\sigma^{\prime}=\operatorname{Int}(v s \theta(v)) \circ \theta$. Then,

$$
\Delta_{\theta}\left(\sigma^{\prime}\right)=\left(\operatorname{Nrp}_{\theta}(v s \theta(v))\right)_{2} \cup[A] .
$$

By $[6,(2.13)], \operatorname{Nrp}_{\theta}(v s \theta(v))=\operatorname{Nrd}_{A}(v) \operatorname{Nrp}_{\theta}(s)$. Since $\left(\operatorname{Nrd}_{A}(v)\right)_{2} \cup[A]=0$, it follows that

$$
\Delta_{\theta}\left(\sigma^{\prime}\right)=\Delta_{\theta}(\sigma)
$$

Similarly, if $\theta^{\prime}$ is a symplectic involution conjugate to $\theta$, then $\Delta_{\sigma^{\prime}}\left(\theta^{\prime}\right)=$ $\Delta_{\sigma^{\prime}}(\theta)$. Now, Part (b) of Proposition 9 shows that $\Delta_{\theta^{\prime}}\left(\sigma^{\prime}\right)=\Delta_{\sigma^{\prime}}\left(\theta^{\prime}\right)$ and $\Delta_{\theta}\left(\sigma^{\prime}\right)=\Delta_{\sigma^{\prime}}(\theta)$. Therefore,

$$
\Delta_{\theta^{\prime}}\left(\sigma^{\prime}\right)=\Delta_{\theta}\left(\sigma^{\prime}\right)
$$

We already observed that $\Delta_{\theta}\left(\sigma^{\prime}\right)=\Delta_{\theta}(\sigma)$, hence

$$
\Delta_{\theta^{\prime}}\left(\sigma^{\prime}\right)=\Delta_{\theta}(\sigma)
$$

and the proof of Proposition 1 is complete. 


\section{Discriminant and trace form.}

In this section we prove Theorem 4 and Proposition 6 .

Let Fields $s_{F}$ be the category of fields containing $F$ and let $G$ be any algebraic group over $F$. We consider the functor

$$
H^{1}(G): \text { Fields }_{F} \rightarrow \text { Sets }^{*}
$$

where Sets* denotes the category of pointed sets, associating to every $L \in$ Fields $s_{F}$ the Galois cohomology set $H^{1}(L, G)$.

For any integer $d \geq 0$, we let $\mathbb{Q} / \mathbb{Z}(d-1)=\lim _{\longrightarrow} \mu_{n}^{\otimes(d-1)}$, where $\mu_{n}$ is the group of $n$-th roots of unity in a separable closure of $F$. We may then consider the functor

$$
H^{d}(\mathbb{Q} / \mathbb{Z}(d-1)): \text { Fields }_{F} \rightarrow \text { Sets }^{*}
$$

which carries $L \in$ Fields $_{F}$ to the Galois cohomology group $H^{d}(L, \mathbb{Q} / \mathbb{Z}(d-1))$ (and forgets the group structure). The natural transformations $H^{1}(G) \rightarrow$ $H^{d}(\mathbb{Q} / \mathbb{Z}(d-1))$ are called cohomological invariants of dimension (or degree $) d$ in $[6, \S 31 . \mathrm{B}]$. Since $H^{d}(L, \mathbb{Q} / \mathbb{Z}(d-1))$ is a group for $L \in$ Fields $_{F}$, these invariants form a group. In reference to Rost's "cohomological cycle module" $M=\bigoplus_{d \geq 0} H^{d}(\bullet, \mathbb{Q} / \mathbb{Z}(d-1)$ ) (see [8]), we denote it simply by $\operatorname{Inv}^{d}\left(H^{1}(G), M\right)$. (This group is denoted by $\operatorname{Inv}^{d}(G, \mathbb{Q} / \mathbb{Z}(d-1))$ in $[\mathbf{6}$, $\S 31 . \mathrm{B}]$.

Now let $A$ be a central simple $F$-algebra of degree $n=2 m$ and let $\theta$ be a symplectic involution on $A$. We take for $G$ the group $\operatorname{GSp}(A, \theta)$ of symplectic similitudes; this is the algebraic group scheme defined by

$$
\mathbf{G S p}(A, \theta)(E)=\left\{g \in A \otimes_{F} E \mid \theta(g) g \in E^{\times}\right\}
$$

for any commutative $F$-algebra $E$. The set $H^{1}(L, \operatorname{GSp}(A, \theta))$ is in oneto-one correspondence with the set of conjugacy classes of symplectic involutions defined on $A_{L}=A \otimes_{F} L$, the class of $\theta$ being the distinguished one (see $[6,(29.23)])$. The following proposition shows that for symplectic involutions there is no (nontrivial) cohomological invariant of degree 1 or 2 .

Proposition 10. If the algebra $A$ is split, we have $H^{1}(L, \mathbf{G S p}(A, \theta))=1$ for every $L \in$ Fields $_{F}$, so that

$$
\operatorname{Inv}^{d}\left(H^{1}(\mathbf{G S p}(A, \theta)), M\right)=0 \quad \text { for all } d .
$$

If $A$ is not split, we have

$$
\operatorname{Inv}^{d}\left(H^{1}(\mathbf{G S p}(A, \theta)), M\right)=0 \quad \text { for } d=1,2
$$

and

$$
\operatorname{Inv}^{3}\left(H^{1}(\mathbf{G S p}(A, \theta)), M\right)=\left\{\begin{array}{lll}
0 & \text { if } \operatorname{deg} A \equiv 2 & \bmod 4 \\
\mathbb{Z} / 2 \mathbb{Z} & \text { if } \operatorname{deg} A \equiv 0 & \bmod 4
\end{array}\right.
$$


Proof. Every symplectic involution on a split algebra is hyperbolic. Therefore, when $A$ is split, $H^{1}(L, \mathbf{G S p}(A, \theta))=1$ for all $L \in$ Fields $_{F}$.

For the rest of the proof, we may thus assume that $A$ is not split. Let

$$
\mu: \operatorname{GSp}(A, \theta) \rightarrow \mathbf{G}_{\mathbf{m}}
$$

be the homomorphism which associates to each similitude $g$ its multiplier $\mu(g)=\theta(g) g$. The cohomology sequence induced by the exact sequence

$$
1 \rightarrow \mathbf{S p}(A, \theta) \rightarrow \mathbf{G S p}(A, \theta) \stackrel{\mu}{\rightarrow} \mathbf{G}_{\mathbf{m}} \rightarrow 1
$$

yields for every $L \in$ Fields ${ }_{F}$ the exact sequence

$$
L^{\times} \rightarrow H^{1}(L, \mathbf{S p}(A, \theta)) \rightarrow H^{1}(L, \mathbf{G S p}(A, \theta)) \rightarrow 1
$$

since $H^{1}\left(L, \mathbf{G}_{\mathbf{m}}\right)=1$ by Hilbert's Theorem 90 . Therefore, for every $d$, we have an exact sequence

$0 \rightarrow \operatorname{Inv}^{d}\left(H^{1}(\mathbf{G S p}(A, \theta)), M\right) \rightarrow \operatorname{Inv}^{d}\left(H^{1}(\mathbf{S p}(A, \theta)), M\right) \rightarrow \operatorname{Inv}^{d}\left(\mathbf{G}_{\mathbf{m}}, M\right)$.

For $d=1$ or 2 , we obtain, by $[\mathbf{6},(31.15)], \operatorname{Inv}^{d}\left(H^{1}(\mathbf{S p}(A, \theta)), M\right)=0$ and hence

$$
\operatorname{Inv}^{d}\left(H^{1}(\mathbf{G S p}(A, \theta)), M\right)=0 .
$$

The group $\operatorname{Inv}^{3}\left(H^{1}(\mathbf{S p}(A, \theta)), M\right)$ is of order 2, the nontrivial element being the Rost invariant $\Delta_{\theta}$ defined in the introduction. Equation (2) shows that this invariant is zero in $\operatorname{Inv}^{3}\left(\mathbf{G}_{\mathbf{m}}, M\right)$ if and only if $\operatorname{deg} A \equiv 2 \bmod 4$.

When $\operatorname{deg} A \equiv 0 \bmod 4$, the unique nontrivial invariant of degree 3 is the discriminant. Our next goal is to give an explicit description of this invariant in terms of trace forms.

Let $T_{\theta}^{+}: \operatorname{Sym}(A, \theta) \rightarrow F$ be the quadratic form

$$
T_{\theta}^{+}(x)=\operatorname{Trd}_{A}(\theta(x) x)=\operatorname{Trd}_{A}\left(x^{2}\right) .
$$

This forms only depends, up to isometry, on the conjugacy class of $\theta$ since, if $\theta^{\prime}=\operatorname{Int}(v) \circ \theta \circ \operatorname{Int}(v)^{-1}$ for some $v \in A^{\times}$, then $\operatorname{Int}(v)$ defines an isometry between $T_{\theta}^{+}$and $T_{\theta^{\prime}}^{+}$. Consider $L \in$ Fields $s_{F}$. The map sending every symplectic involution $\sigma: A_{L} \rightarrow A_{L}$ to the discriminant

$$
\operatorname{disc}\left(T_{\sigma}^{+}-T_{\theta}^{+}\right) \in L^{\times} / L^{\times 2}=H^{1}\left(L, \mu_{2}\right)
$$

defines a cohomological invariant $H^{1}(\mathbf{G S p}(A, \theta)) \rightarrow H^{1}\left(\mu_{2}\right)$. By Proposition 10, this invariant is trivial, hence $T_{\sigma}^{+}-T_{\theta}^{+} \in I^{2} L$. Similarly, the map sending every symplectic involution $\sigma$ to the Witt (-Clifford) invariant

$$
e_{2}\left(T_{\sigma}^{+}-T_{\theta}^{+}\right) \in H^{2}\left(L, \mu_{2}\right)
$$

defines a cohomological invariant of degree 2. Again, by Proposition 10, we get $e_{2}\left(T_{\sigma}^{+}-T_{\theta}^{+}\right)=0$, and hence $T_{\sigma}^{+}-T_{\theta}^{+} \in I^{3} L$ using Merkurjev's theorem. This proves the first part of Theorem 4. Note that the equality 
$e_{2}\left(T_{\sigma}^{+}-T_{\theta}^{+}\right)=0$ can also be derived from Quéguiner's explicit calculation of the Hasse invariant of trace forms in [7, p. 307].

Consider the map associating to every symplectic involution $\sigma: A_{L} \rightarrow A_{L}$ the Arason invariant

$$
e_{3}\left(T_{\sigma}^{+}-T_{\theta}^{+}\right) \in H^{3}\left(L, \mu_{2}\right) .
$$

Using Proposition 10, we see that this invariant is trivial if $\operatorname{deg} A \equiv 2 \bmod 4$ or if $A$ is split. We claim that it coincides with the discriminant $\Delta_{\theta}(\sigma)$ if $A$ is nonsplit and $\operatorname{deg} A \equiv 0 \bmod 4$. To prove this, it suffices to show that it is nontrivial because, by Proposition 10, there is a unique nontrivial invariant in $\operatorname{Inv}^{3}\left(H^{1}(\mathbf{G} \mathbf{S p}(A, \theta)), M\right)$. Therefore, our goal is to find a field $L \in$ Fields $s_{F}$ and two symplectic involutions $\sigma_{1}, \sigma_{2}$ on $A_{L}$ such that $e_{3}\left(T_{\sigma_{1}}^{+}-\right.$ $\left.T_{\sigma_{2}}^{+}\right) \neq 0$. If $\theta$ is any involution on $A$, the equality

$$
e_{3}\left(T_{\sigma_{1}}^{+}-T_{\theta}^{+}\right)=e_{3}\left(T_{\sigma_{1}}^{+}-T_{\sigma_{2}}^{+}\right)+e_{3}\left(T_{\sigma_{2}}^{+}-T_{\theta}^{+}\right)
$$

shows that at least one of the terms $e_{3}\left(T_{\sigma_{1}}^{+}-T_{\theta}^{+}\right)$and $e_{3}\left(T_{\sigma_{2}}^{+}-T_{\theta}^{+}\right)$is nonzero, hence the invariant $\sigma \mapsto e_{3}\left(T_{\sigma}^{+}-T_{\theta}^{+}\right)$is nontrivial.

After scalar extension to the function field of a suitable generalized SeveriBrauer variety (see [2]), we may assume that ind $A=2$ i.e., that $A$ is Brauerequivalent to a quaternion division algebra $Q$ over $F$. Then, denoting by $V$ an $F$-vector space of dimension $m$, we obtain

$$
A \simeq Q \otimes_{F} \operatorname{End}_{F} V .
$$

For the rest of this section, we fix an isomorphism identifying $A$ with $Q \otimes \operatorname{End}_{F} V$. Let $b$ be a symmetric nondegenerate bilinear form on $V$. The symmetric square $S^{2} V$ and the exterior square $\bigwedge^{2} V$ are endowed with symmetric bilinear forms $b^{S^{2}}$ and $b^{\wedge 2}$ respectively, defined by

$$
b^{S^{2}}\left(x_{1} \cdot x_{2}, y_{1} \cdot y_{2}\right)=b\left(x_{1}, y_{1}\right) b\left(x_{2}, y_{2}\right)+b\left(x_{1}, y_{2}\right) b\left(x_{2}, y_{1}\right)
$$

and

$$
b^{\wedge 2}\left(x_{1} \wedge x_{2}, y_{1} \wedge y_{2}\right)=b\left(x_{1}, y_{1}\right) b\left(x_{2}, y_{2}\right)-b\left(x_{1}, y_{2}\right) b\left(x_{2}, y_{1}\right) .
$$

Lemma 11. Let $Q=(\alpha, \beta)_{F}$. On $A=Q \otimes_{F} \operatorname{End}_{F} V$, consider the symplectic involution $\sigma=\gamma \otimes \mathrm{ad}_{b}$, where $\gamma$ is the quaternion conjugation on $Q$ and $\operatorname{ad}_{b}$ is the (orthogonal) involution adjoint to $b$. Then, the bilinear form $B_{\sigma}^{+}(x, y)=\operatorname{Trd}_{A}(x y)$ on $\operatorname{Sym}(A, \sigma)$ (which is the polar form of the quadratic form $\left.T_{\sigma}^{+}\right)$decomposes as an orthogonal sum

$$
B_{\sigma}^{+}=b^{S^{2}} \perp\langle-\alpha,-\beta, \alpha \beta\rangle \cdot b^{\wedge 2} .
$$

Proof. Let $\operatorname{Skew}\left(\operatorname{End}_{F} V, \operatorname{ad}_{b}\right)$ be the $F$-vector space of endomorphisms $f$ of $V$ such that $\operatorname{ad}_{b}(f)=-f$, and let $Q^{0}$ be the $F$-vector space of pure quaternions in $Q$. A straightforward calculation shows that the decomposition

$$
\operatorname{Sym}(A, \sigma)=\left(F \otimes \operatorname{Sym}\left(\operatorname{End}_{F} V, \operatorname{ad}_{b}\right)\right) \oplus\left(Q^{0} \otimes \operatorname{Skew}_{\left.\left(\operatorname{End}_{F} V, \operatorname{ad}_{b}\right)\right)}\right.
$$


is orthogonal with respect to the form $B_{\sigma}^{+}$. Let $B_{b}^{+}$and $B_{b}^{-}$be the restrictions of the bilinear trace form $B(f, g)=\operatorname{tr}(f g)$ to $\operatorname{Sym}\left(\operatorname{End}_{F} V, \operatorname{ad}_{b}\right)$ and Skew $\left(\operatorname{End}_{F} V, \operatorname{ad}_{b}\right)$ respectively. The decomposition above yields

$$
B_{\sigma}^{+}=\langle 2\rangle \cdot B_{b}^{+} \perp\langle 2 \alpha, 2 \beta,-2 \alpha \beta\rangle \cdot B_{b}^{-} .
$$

The lemma follows, since, by $[\mathbf{6},(11.4)] B_{b}^{+} \simeq \frac{1}{2} b^{S^{2}}$ and $B_{b}^{-} \simeq-\frac{1}{2} b^{\wedge 2}$.

If $b=\left\langle a_{1}, \ldots, a_{m}\right\rangle$ is a diagonalization of $b$, it is easily verified that

$$
b^{S^{2}} \simeq m\langle 2\rangle \perp\left(\perp_{i<j}\left\langle a_{i} a_{j}\right\rangle\right) \quad \text { and } \quad b^{\wedge 2} \simeq \perp_{i<j}\left\langle a_{i} a_{j}\right\rangle
$$

(cf $[6$, p. 135]). The formula of the preceding lemma can then be written as

$$
B_{\sigma}^{+} \simeq m\langle 2\rangle \perp\langle 1,-\alpha,-\beta, \alpha \beta\rangle \cdot b^{\wedge 2},
$$

hence, in terms of quadratic forms,

$$
T_{\sigma}^{+}=m\langle 2\rangle+n_{Q} \cdot q^{\wedge 2}
$$

where $n_{Q}$ denotes the norm form of $Q$ and $q^{\wedge 2}$ is the quadratic form defined by $q^{\wedge 2}(x):=b^{\wedge 2}(x, x)$.

Let $b_{1}$ and $b_{2}$ be two nonsingular symmetric bilinear forms on $V$, and let

$$
\sigma_{1}=\gamma \otimes \operatorname{ad}_{b_{1}}, \quad \sigma_{2}=\gamma \otimes \operatorname{ad}_{b_{2}}
$$

be the symplectic involutions on $A=Q \otimes \operatorname{End}_{F} V$ constructed as in the preceding lemma. Observe that $T_{\sigma_{1}}^{+}-T_{\sigma_{2}}^{+}=n_{Q} \cdot\left(q_{1}^{\wedge 2}-q_{2}^{\wedge 2}\right)$, hence

$$
e_{3}\left(T_{\sigma_{1}}^{+}-T_{\sigma_{2}}^{+}\right)=[Q] \cup \operatorname{disc}\left(q_{1}^{\wedge 2}-q_{2}^{\wedge 2}\right) .
$$

Explicit calculation shows that

$$
\operatorname{disc}\left(q_{1}^{\wedge 2}-q_{2}^{\wedge 2}\right)=\operatorname{det} b_{1}^{\wedge 2} \cdot \operatorname{det} b_{2}^{\wedge 2}=\left(\operatorname{det} b_{1} \cdot \operatorname{det} b_{2}\right)^{m-1} .
$$

Adjoining an indeterminate to $F$ if necessary, we may assume that there exists an element $t \in F^{\times}$not belonging to $\operatorname{Nrd}(Q)$. By a theorem of Merkurjev, this element satisfies $[Q] \cup(t)_{2} \neq 0$. It is easy to find two bilinear forms $b_{1}$ and $b_{2}$ on $V$ such that $\operatorname{det} b_{1} \cdot \operatorname{det} b_{2}=t F^{\times 2}$. Since $m$ is even, it follows from (4) and (5) that the corresponding involutions $\sigma_{1}$ and $\sigma_{2}$ satisfy

$$
e_{3}\left(T_{\sigma_{1}}^{+}-T_{\sigma_{2}}^{+}\right) \neq 0
$$

This completes the proof of Theorem 4 .

We now turn to Proposition 6 and assume $A=M_{2}(F) \otimes_{F} A_{0}$. Since all hyperbolic involutions are conjugate, we may assume moreover $\theta=\gamma \otimes \theta_{0}$ for some orthogonal involution $\theta_{0}$ on $A_{0}$, where $\gamma$ is the unique symplectic involution on $M_{2}(F)$ (which is hyperbolic). As in Lemma 11, we have an orthogonal decomposition

$$
\operatorname{Sym}(A, \theta)=\left(F \otimes \operatorname{Sym}\left(A_{0}, \theta_{0}\right)\right) \oplus\left(\operatorname{Skew}\left(M_{2}(F), \gamma\right) \otimes \operatorname{Skew}\left(A_{0}, \theta_{0}\right)\right)
$$

which yields

$$
T_{\sigma}^{+}=\langle 2\rangle \cdot T_{\theta_{0}}^{+} \perp\langle 2,-2,-2\rangle \cdot T_{\theta_{0}}^{-} .
$$


Therefore, $T_{\sigma}^{+}$is Witt-equivalent to $\langle 2\rangle \cdot\left(T_{\theta_{0}}^{+}-T_{\theta_{0}}^{-}\right)=\langle 2\rangle \cdot T_{A_{0}}$. Since the adjoint involution to $T_{\theta}$ is $\theta \otimes \theta$, by $[\mathbf{6},(11.1)]$, it is clear that $T_{\theta}$ is hyperbolic when $\theta$ is hyperbolic. If $\operatorname{deg} A \equiv 2 \bmod 4$, then $\operatorname{deg} A_{0}$ is odd, hence $A_{0}$ is split. Therefore, $A$ is also split. The other statements in Proposition 6 follow from Corollary 5.

\section{Discriminant and decomposability of involutions.}

Our first goal in this section is to give a proof of Theorem 7. As observed in Section 1.3, the theorem is easy if ind $A=2$. Therefore, we assume ind $A=4$. We may then represent $A$ as

$$
A=\operatorname{End}_{D} V
$$

where $D$ is a division algebra of degree 4 and $V$ is a 2-dimensional $D$-vector space. Let $\theta_{0}$ be an arbitrary symplectic involution on $D$. The involution $\sigma$ is adjoint to a Hermitian form $h$ on $V$ (with respect to $\theta_{0}$ ). Using an orthogonal basis of $V$ relative to $h$, we may identify

$$
A=M_{2}(D) \quad \text { and } \quad \sigma=\operatorname{Int} \operatorname{diag}\left(u_{1}, u_{2}\right) \circ \hat{\theta}_{0}
$$

for some $u_{1}, u_{2} \in \operatorname{Sym}\left(D, \theta_{0}\right)^{\times}$, where

$$
\hat{\theta}_{0}\left(\left(a_{i j}\right)_{1 \leq i, j \leq 2}\right)=\left(\theta_{0}\left(a_{i j}\right)\right)_{1 \leq i, j \leq 2}^{t},
$$

i.e., $\hat{\theta}_{0}=t \otimes \theta$ on $A=M_{2}(F) \otimes_{F} D$. Substituting $\operatorname{Int}\left(u_{1}\right) \circ \theta_{0}$ for $\theta_{0}$, we may assume $u_{1}=1$. By $[6,(16.16)]$, we may find a decomposition of $D$ into a tensor product of quaternion subalgebras stable under $\theta_{0}$,

$$
D=Q_{1} \otimes_{F} Q, \quad \theta_{0}=\theta_{1} \otimes \gamma
$$

where $\theta_{1}$ is an orthogonal involution on $Q_{1}$ and $\gamma$ is the canonical involution on $Q$. Moreover, we may assume $u_{2} \in Q_{1}$. Then

$$
\sigma=\operatorname{Int} \operatorname{diag}\left(1, u_{2}\right) \circ\left(t \otimes \theta_{1} \otimes \gamma\right)=\sigma_{0} \otimes \gamma
$$

with $\sigma_{0}=\operatorname{Int} \operatorname{diag}\left(1, u_{2}\right) \circ t \otimes \theta_{1}$ on $M_{2}(F) \otimes Q_{1}$. Theorem 7 is thus proved. Note that the quaternion algebra $Q$ is not uniquely determined by $[6,(16.16)]$.

Let us now prove Theorem 8 , starting with the following general remark:

Lemma 12. For $i=1,2$, let $A_{i}$ be a central simple $F$-algebra with involutions $\sigma_{i}, \theta_{i}$. Assume:

(a) $\operatorname{deg} A_{1} \equiv 2 \bmod 4$ and $\sigma_{1}, \theta_{1}$ orthogonal, and

(b) $\operatorname{deg} A_{2} \equiv 0 \bmod 4$ and $\sigma_{2}, \theta_{2}$ symplectic.

Then $\Delta_{\theta_{1} \otimes \theta_{2}}\left(\sigma_{1} \otimes \sigma_{2}\right)=0$.

Proof. Consider $u_{1} \in \operatorname{Sym}\left(A_{1}, \theta_{1}\right)^{\times}$and $u_{2} \in \operatorname{Sym}\left(A_{2}, \theta_{2}\right)^{\times}$such that

$$
\sigma_{1}=\operatorname{Int}\left(u_{1}\right) \circ \theta_{1} \quad \text { and } \quad \sigma_{2}=\operatorname{Int}\left(u_{2}\right) \circ \theta_{2},
$$


hence

$$
\sigma_{1} \otimes \sigma_{2}=\operatorname{Int}\left(u_{1} \otimes u_{2}\right) \circ\left(\theta_{1} \otimes \theta_{2}\right) .
$$

Then $\Delta_{\theta_{1} \otimes \theta_{2}}\left(\sigma_{1} \otimes \sigma_{2}\right)=\left(\operatorname{Nrp}_{\theta_{1} \otimes \theta_{2}}\left(u_{1} \otimes u_{2}\right)\right)_{2} \cup\left[A_{1} \otimes A_{2}\right]$, and Lemma $9(\mathrm{~d})$ yields

$$
\operatorname{Nrp}_{\theta_{1} \otimes \theta_{2}}\left(u_{1} \otimes u_{2}\right)=\operatorname{Nrd}_{A_{1}}\left(u_{1}\right)^{\frac{1}{2} \operatorname{deg} A_{2}} \operatorname{Nrd}_{A_{2}}\left(u_{2}\right)^{\frac{1}{2} \operatorname{deg} A_{1}} .
$$

Since $\operatorname{deg} A_{2} \equiv 0 \bmod 4$, the first factor is a square. Moreover, since $\sigma_{2}$ and $\theta_{2}$ are of symplectic type,

$$
\operatorname{Nrd}_{A_{2}}\left(u_{2}\right)=\operatorname{Nrp}_{\theta_{2}}\left(u_{2}\right)^{2} .
$$

Therefore, Equation (6) shows that $\operatorname{Nrp}_{\theta_{1} \otimes \theta_{2}}\left(u_{1} \otimes u_{2}\right) \in F^{\times 2}$, so that

$$
\Delta_{\theta_{1} \otimes \theta_{2}}\left(\sigma_{1} \otimes \sigma_{2}\right)=0 \text {. }
$$

Even in the case $\operatorname{deg} A=8$, there may be symplectic involutions $\theta, \sigma$ on $A$ which do not decompose as in Lemma 12, even though $\Delta_{\theta}(\sigma)=0$. Indeed, there are examples of algebras with involution which do not contain any invariant quaternion subalgebra on which the restriction of the involution is of orthogonal type. Suppose $\theta$ is a symplectic involution on a central simple algebra $A$ of degree 8 , and $A_{1} \subset A$ is a quaternion subalgebra on which $\theta$ restricts to an orthogonal involution $\theta_{1}$. The restriction of $\theta$ to the centralizer of $A_{1}$ is then symplectic, hence [6, (16.16)] yields a decomposition

$$
(A, \theta)=\left(A_{1}, \theta_{1}\right) \otimes\left(A_{2}, \theta_{2}\right) \otimes\left(A_{3}, \gamma_{3}\right),
$$

where $A_{1}, A_{2}$ and $A_{3}$ are quaternion algebras, $\theta_{1}$ and $\theta_{2}$ are orthogonal involutions on $A_{1}$ and $A_{2}$ respectively, and $\gamma_{3}$ is the canonical involution on $A_{3}$. This implies, in particular, that the signature of $\theta$ with respect to every ordering of the field $F$ is either 0 or 8 . For example, if $F=\mathbb{R}$ is the field of real numbers and $\theta$ is the involution adjoint to the Hermitian form $\langle 1,1,1,-1\rangle$ on the usual quaternion algebra $\mathbb{H}$, then $\operatorname{sgn} \theta=4$, and so $A=$ $M_{4}(\mathbb{H})$ has no quaternion subalgebras on which $\theta$ restricts to an orthogonal involution. Therefore, even though $\Delta_{\theta}(\theta)=0$, there is no decomposition as in Lemma 12. (See Example 13 for a subtler example.)

Returning to the proof of Theorem 8, we suppose until the end of this section that $A$ is a central simple $F$-algebra of degree 8 , with index dividing 4. Let $\sigma$ be a symplectic involution on $A$, and suppose $A_{1} \simeq M_{2}(F)$ is an invariant subalgebra on which the restriction of $\sigma$ is an orthogonal involution. In this situation, we have a decomposition $A=A_{1} \otimes A_{1}^{\prime}$, where $A_{1}^{\prime}$ denotes the centralizer of $A_{1}$, and $\sigma=\sigma_{1} \otimes \sigma_{1}^{\prime}$, where $\sigma_{1}$ and $\sigma_{1}^{\prime}$ are the restrictions of $\sigma$ to $A_{1}$ and $A_{1}^{\prime}$ respectively. As $A_{1} \simeq M_{2}(F)$, we can find a hyperbolic orthogonal involution $\theta_{1}$ on $A_{1}$ and set

$$
\theta=\theta_{1} \otimes \sigma_{1}^{\prime} \text {. }
$$


The involution $\theta$ is hyperbolic of symplectic type and, by Lemma 12, we have

$$
\Delta(\sigma)=\Delta_{\theta}(\sigma)=0
$$

Conversely, let $\sigma$ be a symplectic involution on $A$ such that $\Delta(\sigma)=0$. To prove that $\sigma$ leaves invariant a subalgebra of $A$ isomorphic to $M_{2}(F)$ on which it restricts to an orthogonal involution, we consider separately various cases, depending on the index of $A$. If $A$ is split, every symplectic involution is hyperbolic and the property is a consequence of [1, Theorem 2.2]. If ind $A=2$, we can always represent $A$ in the form

$$
A=\operatorname{End}_{Q} V
$$

where $Q$ is a quaternion algebra and $V$ is a 4-dimensional vector space over $Q$. The involution $\sigma$ is then adjoint to a Hermitian form $h$ on $V$ (with respect to the canonical involution $\gamma$ on $Q$ ). Let $e$ be an orthogonal basis for $h$. Since $h$ is determined by $\sigma$ up to a factor in $F^{\times}$, we may assume that the diagonalization of $h$ with respect to the basis $e$ is $\left\langle 1, \alpha_{1}, \alpha_{2}, \alpha_{3}\right\rangle$ with $\alpha_{1}, \alpha_{2}, \alpha_{3} \in F^{\times}$. Let $V_{0} \subset V$ be the $F$-subspace with basis $e$. We have $V=V_{0} \otimes_{F} Q$ and

$$
A=\left(\operatorname{End}_{F} V_{0}\right) \otimes_{F} Q, \quad \sigma=\sigma_{0} \otimes \gamma
$$

where $\sigma_{0}$ is the orthogonal involution on $\operatorname{End}_{F} V_{0}$ adjoint to the bilinear symmetric form $\left\langle 1, \alpha_{1}, \alpha_{2}, \alpha_{3}\right\rangle$. As in Example 2, $\Delta(\sigma)=\left(\alpha_{1} \alpha_{2} \alpha_{3}\right)_{2} \cup[Q]$. Therefore, the condition $\Delta(\sigma)=0$ implies, by a theorem of Merkurjev, that $\alpha_{1} \alpha_{2} \alpha_{3} \in \operatorname{Nrd}_{Q}\left(Q^{\times}\right)$. Changing basis if necessary, we may assume that $\alpha_{3}=\alpha_{1} \alpha_{2}$. Then

$$
\left\langle 1, \alpha_{1}, \alpha_{2}, \alpha_{3}\right\rangle=\left\langle 1, \alpha_{1}\right\rangle \otimes\left\langle 1, \alpha_{2}\right\rangle .
$$

This implies $\sigma_{0}=\sigma_{1} \otimes \sigma_{2}$ on $\operatorname{End}_{F} V_{0} \simeq M_{2}(F) \otimes M_{2}(F)$, where $\sigma_{1}$ and $\sigma_{2}$ are the involutions adjoint to the bilinear forms $\left\langle 1, \alpha_{1}\right\rangle$ and $\left\langle 1, \alpha_{2}\right\rangle$, respectively. This proves the theorem in this case.

Finally, suppose ind $A=4$. As in the proof of Theorem 7 given at the beginning of this section, we may then represent $A$ as $A=\operatorname{End}_{D} V$ where $D$ is a division algebra of degree 4 and $V$ is a 2-dimensional $D$-vector space. For the rest of the proof, we use the same notation as in the proof of Theorem 7 . We may thus assume $A=M_{2}(D)=M_{2}(F) \otimes_{F} D$ and $\sigma=\operatorname{Int} \operatorname{diag}\left(1, u_{2}\right) \circ \hat{\theta}_{0}$ for some symplectic involution $\theta_{0}$ on $D$ and $\hat{\theta}_{0}=t \otimes \theta_{0}$. The involution $\theta=\operatorname{Int} \operatorname{diag}(1,-1) \circ \hat{\theta}_{0}$ is hyperbolic, and $\sigma=\operatorname{Int} \operatorname{diag}\left(1,-u_{2}\right) \circ \theta$. By Lemma $9(\mathrm{e})$, we have

$$
\operatorname{Nrp}_{\theta}\left(\operatorname{diag}\left(1,-u_{2}\right)\right)=\operatorname{Nrp}_{\theta_{0}}\left(-u_{2}\right)=\operatorname{Nrp}_{\theta_{0}}\left(u_{2}\right),
$$

hence

$$
\Delta(\sigma)=\Delta_{\theta}(\sigma)=\left(\operatorname{Nrp}_{\theta_{0}}\left(u_{2}\right)\right)_{2} \cup[D] .
$$


Therefore, the condition $\Delta(\sigma)=0$ implies by $[6,(16.19)]$ that the involution $\operatorname{Int}\left(u_{2}\right) \circ \theta_{0}$ on $D$ is conjugate to $\theta_{0}$. We may then find $v \in D^{\times}$such that

$$
\operatorname{Int}\left(u_{2}\right) \circ \theta_{0}=\operatorname{Int}(v) \circ \theta_{0} \circ \operatorname{Int}(v)^{-1}=\operatorname{Int}\left(v \theta_{0}(v)\right) \circ \theta_{0},
$$

hence $u_{2}=v \theta_{0}(v) \lambda$ for some $\lambda \in F^{\times}$. The involution $\sigma$ is conjugate to

$$
\operatorname{Int} \operatorname{diag}(1, v)^{-1} \circ \sigma \circ \operatorname{Int} \operatorname{diag}(1, v)=\operatorname{Int} \operatorname{diag}(1, \lambda) \circ \hat{\theta}_{0},
$$

which restricts to $\operatorname{Int} \operatorname{diag}(1, \lambda) \circ t$ on $M_{2}(F) \subset M_{2}(D)$. Therefore, $\sigma$ leaves the subalgebra $A_{1}=\operatorname{diag}(1, v) M_{2}(F) \operatorname{diag}\left(1, v^{-1}\right)$ invariant and restricts to an orthogonal involution $\sigma_{1}$ on that subalgebra. We thus have a decomposition

$$
(A, \sigma)=\left(A_{1}, \sigma_{1}\right) \otimes\left(A_{1}^{\prime}, \sigma_{1}^{\prime}\right)
$$

where $A_{1}^{\prime}$ is the centralizer of $A_{1}$ and $\sigma_{1}^{\prime}$ is the restriction of $\sigma$ to $A_{1}^{\prime}$. The involution $\sigma_{1}^{\prime}$ is symplectic, hence, by $[\mathbf{6},(16.16)]$, there is a decomposition

$$
\left(A_{1}^{\prime}, \sigma_{1}^{\prime}\right)=\left(A_{2}, \sigma_{2}\right) \otimes\left(A_{3}, \gamma_{3}\right) .
$$

The proof of Theorem 8 is thus complete.

Example 13. The following is another example where the discriminant vanishes even though there is no decomposition as in Lemma 12. Consider three quadratic extensions $K_{1}, K_{2}, K_{3}$ of a field $k$,

$$
K_{i}=k\left(\sqrt{a_{i}}\right) \quad \text { for some } a_{i} \in k,
$$

such that $K_{1} \otimes_{k} K_{2} \otimes_{k} K_{3}$ is a field, and let $F=k\left(x_{1}, x_{2}, x_{3}\right)$ be the field of rational fractions in three indeterminates over $k$. For $i=1,2,3$, consider $K_{i}$ as a subfield of the quaternion algebra $A_{i}=\left(a_{i}, x_{i}\right)_{F}$. On the tensor product

$$
A=A_{1} \otimes_{F} A_{2} \otimes_{F} A_{3},
$$

consider the symplectic involution

$$
\theta=\theta_{1} \otimes \theta_{2} \otimes \gamma_{3},
$$

where $\gamma_{3}$ is the conjugation involution on $A_{3}$ and $\theta_{1}$ (resp. $\theta_{2}$ ) is an orthogonal involution on $A_{1}$ (resp. $\left.A_{2}\right)$ which is the identity on $K_{1}$ (resp. $K_{2}$ ).

Let $\lambda \in N_{K_{1} / k}\left(K_{1}^{\times}\right) \cap N_{K_{2} / k}\left(K_{2}^{\times}\right)$. By a well-known property of biquadratic extensions (see for instance [4, 2.13]), we may find $u \in K_{1} \otimes_{k} K_{2}$ and $v \in k^{\times}$such that

$$
\lambda=v^{2} N_{K_{1} \otimes_{k} K_{2} / k}(u) .
$$

Viewing $u=u \otimes 1$ as an element of $A$, we let

$$
\sigma=\operatorname{Int}(u) \circ \theta \text {. }
$$

By Lemma 9(d), we have $\operatorname{Nrp}_{\theta}(u)=\operatorname{Nrd}_{A_{1} \otimes_{F} A_{2}}(u)=N_{K_{1} \otimes_{k} K_{2} / k}(u)$, hence

$$
\Delta_{\theta}(\sigma)=(\lambda) \cup[A] .
$$


Since $\lambda$ is a norm from $K_{1}$ and $K_{2}$, hence a reduced norm from $A_{1}$ and $A_{2}$, it follows that

$$
\Delta_{\theta}(\sigma)=(\lambda) \cup\left[A_{3}\right] .
$$

Hence, $\Delta_{\theta}(\sigma)=0$ if and only if $\lambda \in N_{K_{1} / k}\left(K_{1}^{\times}\right) \cap N_{K_{2} / k}\left(K_{2}^{\times}\right) \cap N_{K_{3} / k}\left(K_{3}^{\times}\right)$.

Now, suppose $\sigma=\operatorname{Int}(w) \circ \sigma^{\prime} \circ \operatorname{Int}(w)^{-1}$ for some involution $\sigma^{\prime}$ leaving $A_{1}$ invariant. Then $\sigma^{\prime}=\operatorname{Int}\left(w^{-1} u \theta(w)^{-1}\right) \circ \theta$, and the proof of Lemma 12 shows that $\operatorname{Nrp}_{\theta}\left(w^{-1} u \theta(w)^{-1}\right) \in F^{\times 2}$. Since

$$
\operatorname{Nrp}_{\theta}\left(w^{-1} u \theta(w)^{-1}\right)=\operatorname{Nrp}_{\theta}(u) \operatorname{Nrd}_{A}(w)^{-1}=\lambda v^{-2} \operatorname{Nrd}_{A}(w)^{-1},
$$

it follows that

$$
\lambda \in F^{\times 2} \cdot \operatorname{Nrd}_{A}\left(A^{\times}\right) .
$$

By Proposition 9 of [3], we then have

$$
\lambda \in k^{\times 2} \cdot N_{M / k}\left(M^{\times}\right) \quad \text { with } M=K_{1} \otimes_{k} K_{2} \otimes_{k} K_{3} .
$$

Therefore, examples of triquadratic extensions $M=K_{1} \otimes_{k} K_{2} \otimes_{k} K_{3} / k$ such that

$$
N_{K_{1} / k}\left(K_{1}^{\times}\right) \cap N_{K_{2} / k}\left(K_{2}^{\times}\right) \cap N_{K_{3} / k}\left(K_{3}^{\times}\right) \neq k^{\times 2} \cdot N_{M / k}\left(M^{\times}\right)
$$

yield examples of involutions $\sigma$ for which $\Delta_{\theta}(\sigma)=0$ even though $\sigma$ is not conjugate to an involution leaving $A_{1}$ invariant. Triquadratic extensions of this type were constructed in [9] (see also [5, Proposition 3]).

\section{References}

[1] E. Bayer-Fluckiger, D.B. Shapiro and J.-P. Tignol, Hyperbolic involutions, Math. Z., 214 (1993), 461-476, MR 94j:16060, Zbl 0796.16029.

[2] A. Blanchet, Function fields of generalized Brauer-Severi varieties, Comm. Algebra, 19 (1991), 97-118, MR 92c:14052, Zbl 0717.16014.

[3] M. Chacron, H. Dherte, J.-P. Tignol, A.R. Wadsworth and V.I. Yanchevskiŭ, Discriminants of involutions on Henselian division algebras, Pacific J. Math., 167 (1995), 49-79, MR 95k:16020, Zbl 0826.16013.

[4] R. Elman and T.Y. Lam, Quadratic forms under algebraic extensions, Math. Ann., 219 (1976), 21-42, MR 53 \#5476, Zbl 0308.10012.

[5] P. Gille, Examples of non-rational varieties of adjoint groups, J. Algebra, 193 (1997), 728-747, MR 98k:11038, Zbl 0911.14026.

[6] M.-A. Knus, A.S. Merkurjev, M. Rost and J.-P. Tignol, The Book of Involutions, Amer. Math. Soc. Coll. Pub., 44, AMS, Providence, RI, 1998, MR 2000a:16031, Zbl 0955.16001.

[7] A. Quéguiner, Cohomological invariants of algebras with involution, J. Algebra, 194 (1997), 299-330, MR 98i:16019, Zbl 0904.16009.

[8] M. Rost, Chow groups with coefficients, Documenta Math., 1 (1996), 319-393 (electronic), MR 98a:14006, Zbl 0864.14002. 
[9] D.B. Shapiro, J.-P. Tignol and A.R. Wadsworth, Witt rings and Brauer groups under multiquadratic extensions II, J. Algebra, 78 (1982), 58-90, MR 85i:11033, Zbl 0492.10015.

Received March 15, 2002 and revised May 20, 2002. The authors thank the referee for his/her careful reading and constructive remarks. They gratefully acknowledge support from the TMR network " $K$-theory and linear algebraic groups" (ERB FMRX CT97-0107). The third author is also grateful to the National Fund for Scientific Research (Belgium) for partial support.

DÉPARTEMENT DE MATHÉMATIQUES

École Polytechnique Fédérale de Lausanne

CH-1015 LAUSANNE

SWITZERLAND

E-mail address: gregory.berhuy@epfl.ch

DÉPARTEMENT DE MATHÉMATIQUES

École Polytechnique FÉdérale de Lausanne

CH-1015 LAUSANNE

SWITZERLAND

E-mail address: marina.monsurro@epfl.ch

Institut de Mathématique Pure et Appliquée

UNIVERSité CATHOLIQUE DE LOUVAIN

B-1348 Louvain-LA-Neuve

BELGIUM

E-mail address: tignol@math.ucl.ac.be 